\title{
Product States for Local Algebras
}

\author{
Detlev Buchholz \\ II. Institut für Theoretische Physik der Universität Hamburg, \\ Hamburg, Federal Republic of Germany
}

Received February 8, 1974

\begin{abstract}
Let $\mathscr{R}\left(\mathcal{O}_{1}\right) \subset \mathscr{B}(\mathscr{H})$ and $\mathscr{R}\left(\mathcal{O}_{2}\right) \subset \mathscr{B}(\mathscr{H})$ be the von Neumann algebras associated with the space-time regions $\mathcal{O}_{1}$ and $\mathcal{O}_{2}$ respectively in the vacuum representation of the free neutral massive scalar field. For suitably chosen spacelike separated regions $\mathcal{O}_{1}$ and $\mathcal{O}_{2}$ it is proved that there exists a normal product state $\varphi$ of $\mathscr{B}(\mathscr{H})$,

$$
\varphi(A B)=\varphi(A) \cdot \varphi(B) \text { for all } A \in \mathscr{R}\left(\mathcal{O}_{1}\right) \text { and } B \in \mathscr{R}\left(\mathcal{O}_{2}\right) .
$$
\end{abstract}

Some consequences for the algebraic structure of the local rings are pointed out.

\section{Introduction}

It has been shown by Roos [1] that the local $C^{*}$-algebras $\mathfrak{A}\left(\mathcal{O}_{1}\right)$ and $\mathfrak{A}\left(\mathcal{O}_{2}\right)$ associated with spacelike separated regions $\mathcal{O}_{1}$ and $\mathcal{O}_{2}$ in Minkowski space are statistically independent: every pair of states $\varphi_{1}$ of $\mathfrak{U}\left(\mathcal{O}_{1}\right)$ and $\varphi_{2}$ of $\mathfrak{A}\left(\mathcal{O}_{2}\right)$ can be extended to a state $\varphi$ of $\mathfrak{A}$ (the $C^{*}$-algebra generated by all local $C^{*}$-algebras). Moreover, $\varphi$ can be chosen to be a product state for $\mathfrak{H}\left(\mathcal{O}_{1}\right)$ and $\mathfrak{A}\left(\mathcal{O}_{2}\right)$ :

$$
\varphi(A B)=\varphi(A) \cdot \varphi(B) \quad \text { for all } A \in \mathfrak{A}\left(\mathcal{O}_{1}\right) \text { and } B \in \mathfrak{A}\left(\mathcal{O}_{2}\right) .
$$

For a general structure analysis of physically interesting representations of the local $C^{*}$-algebras it would be important to know whether one can extend the result of Roos in the following way: let $\mathscr{R}\left(\mathcal{O}_{1}\right)$ and $\mathscr{R}\left(\mathcal{O}_{2}\right)$ be von Neumann sub-algebras of $\mathscr{B}(\mathscr{H})^{1}$ associated with the regions $\mathcal{O}_{1}$ and $\mathcal{O}_{2}$. Take any pair of normal states $\varphi_{1}$ of $\mathscr{R}\left(\mathcal{O}_{1}\right)$ and $\varphi_{2}$ of $\mathscr{R}\left(\mathcal{O}_{2}\right)$; does there exist a normal state $\varphi$ of $\mathscr{B}(\mathscr{H})$ which is an extension of $\varphi_{1}$ and $\varphi_{2}$ and a product state for $\mathscr{R}\left(\mathcal{O}_{1}\right)$ and $\mathscr{R}\left(\mathcal{O}_{2}\right)$ ? If this question had a positive answer the local rings would have the following remarkable properties:

a) From the existence of normal product states one could conclude that $\mathscr{R}\left(\mathscr{R}\left(\mathcal{O}_{1}\right), \mathscr{R}\left(\mathcal{O}_{2}\right)\right)$ (the von Neumann algebra generated by $\mathscr{R}\left(\mathcal{O}_{1}\right)$ and $\left.\mathscr{R}\left(\mathcal{O}_{2}\right)\right)$ is isomorphic to the $W^{*}$-tensor product of $\mathscr{R}\left(\mathcal{O}_{1}\right)$ and $\mathscr{R}\left(\mathcal{O}_{2}\right)$. Locality would reflect itself in a very simple algebraic structure of $\mathscr{R}\left(\mathscr{R}\left(\mathcal{O}_{1}\right), \mathscr{R}\left(\mathcal{O}_{2}\right)\right)$.

\footnotetext{
${ }^{1} \mathscr{\mathscr { B }}(\mathscr{H})$ is the algebra of all bounded operators in the representation space $\mathscr{H}$ of $\mathfrak{Q}$.
} 
b) There is an old conjecture of Borchers which says that it should be possible to embed the local rings $\mathscr{R}\left(\mathcal{O}_{1}\right)$ and $\mathscr{R}\left(\mathcal{O}_{2}\right)$ (which are in general not Type I) into factors of Type I $\mathscr{M}_{1}$ and $\mathscr{M}_{2}$ respectively without loss of the local structure:

$$
\mathscr{R}\left(\mathcal{O}_{1}\right) \subset \mathscr{M}_{1} \subset \mathscr{M}_{2}^{\prime} \subset \mathscr{R}\left(\mathcal{O}_{2}\right)^{\prime} .
$$

With the above extension theorem one could immediately verify this hypothesis. (See the following chapter.)

c) Let $\mathcal{O}_{\alpha}$ and $\mathcal{O}_{\beta}$ be two regions contained in $\mathcal{O}_{1}$. Then it follows from the properties of the local rings quoted above that every isomorphism $\Phi$ which maps the ring $\mathscr{R}\left(\mathcal{O}_{\alpha}\right)$ onto $\mathscr{R}\left(\mathcal{O}_{\beta}\right)$ can be implemented by an unitary operator taken from $\mathscr{M}_{1}$. Hence, $\Phi$ can be extended to an isomorphism which acts trivially on $\mathscr{R}\left(\mathcal{O}_{2}\right)$.

These few remarks may suffice to make plain the relevance of such an extension theorem. Unfortunately we have not been able to prove it starting only from basic principles of quantum field theory. But we shall show that the theorem holds at least in the simple model of the free neutral massive scalar field. So the main purpose of this paper is - besides adding some new information about the good old free field - to show that the usually accepted postulates of field theory are compatible with the existence of normal product states within physically interesting representations of the local algebras.

\section{Some General Remarks}

In this chapter we shall make some more or less incoherent remarks about normal product states. To simplify the discussion we shall restrict our attention to the vacuum representation of the local algebras. We base our arguments on the assumption that the vector $\Omega$ (which represents the vacuum) is cyclic and separating for the von Neumann algebras $\mathscr{R}(\mathcal{O})$ belonging to open regions $\mathcal{O}$ with non-empty spacelike complement $\mathcal{O}^{\prime}$. This Reeh-Schlieder property of the vacuum can be derived from the basic principles of quantum field theory [2]. Furthermore we use the fact that the local rings are "almost" factors [3]: let $\hat{\mathcal{O}}$ be any region containing $\mathcal{O}+\mathscr{N}\left(\mathcal{N}\right.$ a suitably chosen neighbourhood of 0 in $\left.\mathbb{R}^{4}\right)$. Then $A \cdot B=0$ for $A \in \mathscr{R}(\mathcal{O})$ and $B \in \mathscr{R}(\hat{\mathcal{O}})^{\prime}$ implies $A=0$ or $B=0$.

The following simplified version of Theorem 2.7.9 taken from the book of Sakai on $C^{*}$ - and $W^{*}$-algebras [4] will be repeatedly used:

Lemma 2.1. Let $\mathscr{U} \subset \mathscr{B}(\mathscr{H})$ be a von Neumann algebra with a cyclic and separating vector and $\mathbb{1} \in \mathscr{U}$. Then every normal state $\varphi$ of $\mathscr{M}$ can be represented by a vector $\xi \in \mathscr{H}: \varphi()=.(\xi, . \xi)$. If $\varphi$ is faithful, $\xi$ can be chosen to be cyclic for $\mathscr{M}$. 
Now we are prepared to prove the following theorem which says that the existence of a product state implies the existence of a very special one. More precisely:

Theorem 2.2. Let $\hat{\mathcal{O}}_{1}$ and $\hat{\mathcal{O}}_{2}$ be two spacelike separated regions such that $\hat{O}_{1}+\mathscr{N} \subset \hat{\mathcal{O}}_{1}$ and $\hat{O}_{2}+\mathscr{N} \subset \hat{\mathcal{O}}_{2}$. If there exists a normal product state $\varphi$ for $\mathscr{R}\left(\hat{\mathcal{O}}_{1}\right)$ and $\mathscr{R}\left(\hat{\mathcal{O}}_{2}\right)$ then there exists a vector $\eta \in \mathscr{H}$ such that

i) $\varphi_{\eta}()=.(\eta, \eta)$ is a product state for $\mathscr{R}\left(\mathcal{O}_{1}\right)$ and $\mathscr{R}\left(\mathcal{O}_{2}\right)$.

ii) The restriction of $\varphi_{\eta}$ to $\mathscr{R}\left(\mathcal{O}_{1}\right)$ and $\mathscr{R}\left(\mathcal{O}_{2}\right)$ coincides with the restriction of $\varphi_{0}$ (the vacuum functional) to these algebras ${ }^{2}$.

iii) $\eta$ is cyclic and separating for $\mathscr{R}\left(\mathscr{R}\left(\mathcal{O}_{1}\right), \mathscr{R}\left(\mathcal{O}_{2}\right)\right.$ ) (the von Neumann algebra generated by $\mathscr{R}\left(\mathcal{O}_{1}\right)$ and $\left.\mathscr{R}\left(\mathcal{O}_{2}\right)\right)$.

Proof. i) Because we are free to replace $\hat{\mathcal{O}}_{1}$ and $\hat{\mathcal{O}}_{2}$ by somewhat smaller regions we may assume that the spacelike complement of $\hat{\mathcal{O}}_{1} \cup \hat{\mathcal{O}}_{2}$ is not empty. Then the vacuum is cyclic and separating for $\mathscr{R}\left(\mathscr{R}\left(\hat{\mathcal{O}}_{1}\right), \mathscr{R}\left(\hat{\mathcal{O}}_{2}\right)\right)$ and we can represent the restriction of the product state $\varphi$ to the algebra $\mathscr{R}\left(\mathscr{R}\left(\hat{\mathcal{O}}_{1}\right), \mathscr{R}\left(\hat{\mathcal{O}}_{2}\right)\right)$ by a vector $\xi \in \mathscr{H}$. (See the lemma above.)

ii) Let $P_{1}, P_{2}$ be the projections onto the closed subspaces $\left[\mathscr{R}\left(\hat{\mathcal{O}}_{1}\right) \xi\right]$ and $\left[\mathscr{R}\left(\hat{\mathcal{O}}_{2}\right) \xi\right]$ of $\mathscr{H}$. It is obvious that $P_{1} \in \mathscr{R}\left(\hat{\mathcal{O}}_{1}\right)^{\prime}$ and $P_{2} \in \mathscr{R}\left(\hat{\mathcal{O}}_{2}\right)^{\prime}$. From the factorisation property of $\xi$ it follows furthermore that

and

$$
\begin{array}{lll}
P_{1} A P_{1}=(\xi, A \xi) \cdot P_{1} & \text { for } & A \in \mathscr{R}\left(\hat{O}_{2}\right) \\
P_{2} B P_{2}=(\xi, B \xi) \cdot P_{2} & \text { for } & B \in \mathscr{R}\left(\hat{O}_{1}\right) .
\end{array}
$$

Thus the state $\psi()=.\left(P_{1} \Omega, \mathrm{P}_{1} \Omega\right) \cdot\left\|P_{1} \Omega\right\|^{-2}$ is again a product state for $\mathscr{R}\left(\hat{\mathcal{O}}_{1}\right)$ and $\mathscr{R}\left(\hat{\mathcal{O}}_{2}\right)$. From the fact that the local rings are "almost" factors and from the cyclicity of the vacuum for the local rings one can easily conclude that $\psi$ is faithful for $\mathscr{R}\left(\mathcal{O}_{1}\right)$. Again from the lemma quoted above it follows then that there exists a cyclic vector $\xi_{c} \in \mathscr{H}$ which represents the restriction of $\psi$ to $\mathscr{R}\left(\mathcal{O}_{1}\right)$. Now we can construct in a canonical way an isometric operator $U_{1} \in \mathscr{R}\left(\mathcal{O}_{1}\right)^{\prime}$ :

$$
U_{1} A \xi_{c}=A \cdot \frac{P_{1} \Omega}{\left\|P_{1} \Omega\right\|} \quad \text { for } \quad A \in \mathscr{R}\left(\mathcal{O}_{1}\right) .
$$

It is evident that the ranges of $P_{1}$ and $U_{1}$ coincide; so we have $U_{1} U_{1}^{*}=P_{1}$. From this and relation (1) we get

$$
U_{1}^{*} A U_{1}=(\xi, A \xi) \cdot \mathbb{1} \quad \text { for } \quad A \in \mathscr{R}\left(\hat{\mathcal{O}}_{2}\right) .
$$

Therefore the state $\psi_{1}()=.\left(U_{1} \Omega, . U_{1} \Omega\right)$ is a product state for $\mathscr{R}\left(\mathcal{O}_{1}\right)$ and $\mathscr{R}\left(\hat{\mathcal{O}}_{2}\right)$ and the restriction of $\psi_{1}$ to $\mathscr{R}\left(\mathcal{O}_{1}\right)$ coincides with the restriction

\footnotetext{
2 This part of the theorem is due to Rinke.
} 
of the vacuum functional $\varphi_{0}$ to this algebra. If one now carries through the whole construction once more starting with $\psi_{1}$ instead of $\varphi$, then one gets a product state for $\mathscr{R}\left(\mathcal{O}_{1}\right)$ and $\mathscr{R}\left(\mathcal{O}_{2}\right)$ which coincides with $\varphi_{0}$ on each algebra separately.

iii) Let $\mathcal{O}_{1, \varepsilon}$ and $\mathcal{O}_{2, \varepsilon}$ be the regions which are generated by the time translated regions $\mathcal{O}_{1}+t$ and $\mathcal{O}_{2}+t$ with $|t|<\varepsilon$ :

$$
\mathcal{O}_{k, \varepsilon}=\bigcup_{|t|<\varepsilon}\left\{\mathcal{O}_{k}+t\right\} \text { for } k=1,2 .
$$

If $\varepsilon$ is small enough it follows immediately from the preceding discussion that there exists a normal product state $\omega$ for $\mathscr{R}\left(\mathcal{O}_{1, \varepsilon}\right)$ and $\mathscr{R}\left(\mathcal{O}_{2, \varepsilon}\right)$ which coincides with the vacuum $\varphi_{0}$ on each algebra separately. With the help of the unitary time translation operators $U(t)$ we can construct another state:

$$
\omega_{h}(.)=\int_{-\varepsilon}^{\varepsilon} d t h(t) \cdot \omega\left(U(t) \cdot U^{-1}(t)\right)
$$

( $h$ is a smooth positive function and $\int_{-\varepsilon}^{\varepsilon} d t h(t)=1$ ). It is obvious that $\omega_{h}$ is a normal product state for $\mathscr{R}\left(\mathcal{O}_{1}\right)$ and $\mathscr{R}\left(\mathcal{O}_{2}\right)$ which coincides with the vacuum on both algebras. Furthermore, $\omega_{h}$ is a faithful state of $\mathscr{R}\left(\mathscr{R}\left(\mathcal{O}_{1}\right), \mathscr{R}\left(\mathcal{O}_{2}\right)\right)$ : let $Q$ be an element of $\mathscr{R}\left(\mathscr{R}\left(\mathcal{O}_{1}\right), \mathscr{R}\left(\mathcal{O}_{2}\right)\right)$ such that $\omega_{h}\left(Q^{*} Q\right)=0$ and therefore $\omega\left(U(t) Q^{*} Q U^{-1}(t)\right)=0$ for $|t|<\varepsilon$. Clearly there exists a vector state $\varphi_{\zeta}$ such that $\lambda \cdot \varphi_{\zeta} \leqq \omega$ for some $\lambda>0$ and from this it follows that $Q \cdot U^{-1}(t) \zeta=0$ for $|t|<\varepsilon$. It is then standard to conclude that $Q=0$ [3]. Thus we know from the lemma that there exists a vector $\eta \in \mathscr{H}$ which is cyclic and separating for $\mathscr{R}\left(\mathscr{R}\left(\mathcal{O}_{1}\right), \mathscr{R}\left(\mathcal{O}_{2}\right)\right)$; the state $\varphi_{\eta}()=.(\eta, . \eta)$ is a product state for $\mathscr{R}\left(\mathcal{O}_{1}\right)$ and $\mathscr{R}\left(\mathcal{O}_{2}\right)$ and the restriction of $\varphi_{\eta}$ to $\mathscr{R}\left(\mathcal{O}_{1}\right)$ and $\mathscr{R}\left(\mathcal{O}_{2}\right)$ coincides with the restriction of $\varphi_{0}$ to these algebras.

It is now easy to show that the existence of one normal product state implies that the extension theorem (which has been indicated in the introduction) holds:

Corollary 2.3. Let $\hat{\mathcal{O}}_{1}, \hat{\mathcal{O}}_{2}$ and $\hat{O}_{1}, \hat{O}_{2}$ be regions as in the theorem above. If there exists a normal product state for $\mathscr{R}\left(\hat{\mathcal{O}}_{1}\right)$ and $\mathscr{R}\left(\hat{\mathcal{O}}_{2}\right)$, then every pair of normal states $\varphi_{1}$ of $\mathscr{R}\left(\mathcal{O}_{1}\right)$ and $\varphi_{2}$ of $\mathscr{R}\left(\mathcal{O}_{2}\right)$ can be extended to a normal product state $\varphi$ of $\mathscr{R}\left(\mathcal{O}_{1}\right)$ and $\mathscr{R}\left(\mathcal{O}_{2}\right)$.

Proof. To begin with we represent $\varphi_{1}$ and $\varphi_{2}$ by vectors $\eta_{1}, \eta_{2} \in \mathscr{H}$. Since the vacuum $\Omega$ is cyclic for $\mathscr{R}\left(\mathcal{O}_{1}\right)$ and $\mathscr{R}\left(\mathcal{O}_{2}\right)$ we can find sequences $A_{n} \in \mathscr{R}\left(\mathcal{O}_{1}\right)$ and $B_{n} \in \mathscr{R}\left(\mathcal{O}_{2}\right)$ such that $\eta_{1}=\mathrm{s}-\lim A_{n} \Omega$ and $\eta_{2}=\operatorname{s-lim} B_{n} \Omega$. Then we take the vector $\eta$ with the properties specified in the theorem above. It is trivial to verify that $s-\lim A_{n} B_{n} \eta=\zeta$ exists and that the product state $(\zeta, . \zeta)$ is an extension of $\varphi_{1}$ and $\varphi_{2}$. 
As another application of Theorem 2.2 we want to prove that the conjecture of Borchers is true if there exist normal product states.

Corollary 2.4. Let $\eta$ be the vector constructed in Theorem 2.2 and let $P_{1}, P_{2}$ be the projections onto $\left[\mathscr{R}\left(\mathcal{O}_{1}\right) \eta\right]$ and $\left[\mathscr{R}\left(\mathcal{O}_{2}\right) \eta\right]$ respectively. If $\mathscr{M}_{1}$ and $\mathscr{M}_{2}$ are the von Neumann algebras generated by $\mathscr{R}\left(\mathcal{O}_{1}\right), P_{2}$ and $\mathscr{R}\left(\mathrm{O}_{2}\right), P_{1}$ respectively, then

i) $\mathscr{M}_{1}$ and $\mathscr{M}_{2}$ are factors of Type I and

ii) $\mathscr{R}\left(\mathcal{O}_{1}\right) \subset \mathscr{M}_{1} \subset \mathscr{M}_{2}^{\prime} \subset \mathscr{R}\left(\mathcal{O}_{2}\right)^{\prime}$.

Proof. i) From relation (1) in the proof of Theorem 2.2 one derives

and

$$
P_{1} a P_{1}=(\eta, a \eta) \cdot P_{1} \quad \text { for } \quad a \in \mathscr{M}_{2}
$$

$$
P_{2} b P_{2}=(\eta, b \eta) \cdot P_{2} \text { for } b \in \mathscr{M}_{1} \text {. }
$$

Thus $\mathscr{M}_{1}$ contains the abelian projection $P_{2}$ and $\mathscr{M}_{2}$ contains the abelian projection $P_{1}{ }^{3}$. It remains to show that $\mathscr{M}_{1}$ and $\mathscr{M}_{2}$ are factors: let $z$ be any projection in the center of $\mathscr{M}_{1}$. From relation (2) it follows that

$$
z P_{2}=P_{2} z P_{2}=(\eta, z \eta) \cdot P_{2}
$$

$z P_{2}$ is again a projection. Hence $(\eta, z \eta)$ must be 0 or 1 . If $z \eta=0$ it follows that $z=0$, because $z \in \mathscr{R}\left(\mathcal{O}_{1}\right)^{\prime} \cap \mathscr{R}\left(\mathcal{O}_{2}\right)^{\prime}$ and $\eta$ is cyclic for $\mathscr{R}\left(\mathscr{R}\left(\mathcal{O}_{1}\right), \mathscr{R}\left(\mathcal{O}_{2}\right)\right)$. If $(\eta, z \eta)=1$ one concludes that $(\mathbb{1}-z) \eta=0$ and from this follows $z=\mathbb{1}$. So the center of $\mathscr{M}_{1}$ contains only trivial projections. Consequently $\mathscr{M}_{1}$ is a factor and the same argument shows that $\mathscr{M}_{2}$ is a factor too.

ii) Since $P_{1} \in \mathscr{R}\left(\mathcal{O}_{1}\right)^{\prime}$ and $P_{2} \in \mathscr{R}\left(\mathcal{O}_{2}\right)^{\prime}$ it remains to show that $P_{1} P_{2}$ $=P_{2} P_{1}$. Take any $A \in \mathscr{R}\left(\mathcal{O}_{1}\right)$ and $B \in \mathscr{R}\left(\mathcal{O}_{2}\right)$. Then

$$
\begin{aligned}
P_{1} P_{2} A B \eta & =P_{1} P_{2} A P_{2} B \eta=(\eta, A \eta) P_{1} B P_{1} \eta=(\eta, A \eta)(\eta, B \eta) \eta \\
& =P_{2} P_{1} B P_{1} A \eta=P_{2} P_{1} A B \eta .
\end{aligned}
$$

Hence $P_{1}$ and $P_{2}$ commute and therefore $\mathscr{M}_{1} \subset \mathscr{M}_{2}^{\prime}$.

It is obvious, but still worth mentioning, that the existence of factors $\mathscr{M}_{1}$ and $\mathscr{M}_{2}$ with the properties specified above implies the existence of normal product states. As another consequence, it follows that certain "local" isomorphisms can be implemented by unitary operators taken from these factors:

Corollary 2.5. Let $\mathcal{O}_{\alpha}$ and $\mathcal{O}_{\beta}$ be two regions contained in $\mathcal{O}_{1}$. If $\Phi$ is an isomorphism which maps $\mathscr{R}\left(\mathcal{O}_{\alpha}\right)$ onto $\mathscr{R}\left(\mathcal{O}_{\beta}\right)$ then $\Phi$ can be implemented by an unitary operator $U \in \mathscr{M}_{1}$ :

$$
\Phi(A)=U A U^{-1} \text { for all } A \in \mathscr{R}\left(\mathcal{O}_{\alpha}\right) .
$$

${ }^{3}$ For the definition of abelian projections and their connection with Type I von Neumann algebras see [4; Chapter 2.2]. 
Proof. Let $P_{1}$ be the projection defined in Corollary 2.4. Clearly, $P_{1} \mathscr{H}$ is invariant under the action of $\mathscr{M}_{1}$. Thus we can consider the induced representation $\pi_{P_{1}}$ of $\mathscr{M}_{1}$ on $P_{1} \mathscr{H}$. Since $\eta \in P_{1} \mathscr{H}$, this representation is faithful and it is easy to verify that $\pi_{P_{1}}\left(\mathscr{M}_{1}\right)=\mathscr{B}\left(P_{1} \mathscr{H}\right)$.

Now $\pi_{P_{1}}\left(\mathscr{R}\left(\mathcal{O}_{\alpha}\right)\right) \subset \pi_{P_{1}}\left(\mathscr{M}_{1}\right)$ and $\pi_{P_{1}}\left(\mathscr{R}\left(\mathcal{O}_{\beta}\right)\right) \subset \pi_{P_{1}}\left(\mathscr{M}_{1}\right)$ both have a cyclic vector, $P_{1} \Omega \in P_{1} \mathscr{H}$, and a separating vector, $\eta \in P_{1} \mathscr{H}$. Hence every isomorphism which maps $\pi_{P_{1}}\left(\mathscr{R}\left(\mathcal{O}_{\alpha}\right)\right)$ onto $\pi_{P_{1}}\left(\mathscr{R}\left(\mathcal{O}_{\beta}\right)\right)$ is spatial $\left[5 ;\right.$ p. 222, Theorem 3]. Thus there exists an unitary operator $V \in \pi_{P_{1}}\left(\mathscr{M}_{1}\right)$ such that

$$
\pi_{P_{1}} \circ \Phi(A)=V \pi_{P_{1}}(A) V^{-1} \text { for all } A \in \mathscr{R}\left(\mathcal{O}_{\alpha}\right)
$$

and from this relation the statement follows immediately.

To conclude this chapter we want to point out that one cannot expect to have normal product states for arbitrary spacelike separated regions $\mathcal{O}_{1}$ and $\mathcal{O}_{2}$. There are essentially two classes of configurations for which such states cannot occur.

a) If the closures of the regions $\mathcal{O}_{1}$ and $\mathcal{O}_{2}$ are not spacelike separated, then, at least for the free field, it is easy to show that one runs into contradictions if one postulates the existence of normal product states for such regions. This "boundary effect" is intimately connected with the infinite extension of momentum space.

b) A typical example of the second class of configurations not admitting normal product states is the following one ${ }^{4}$ : Let $\mathcal{O}_{1}$ and $\mathcal{O}_{2}$ be two spacelike separated regions which are maped into themselves by the translation $a: \mathcal{O}_{1}+a \subseteq \mathcal{O}_{1}$ and $\mathcal{O}_{2}+a \leqq \mathcal{O}_{2}$. From the isotony of the local net it follows then that $\mathscr{R}\left(\mathcal{O}_{1}+n a\right) \subseteq \mathscr{R}\left(\mathcal{O}_{1}\right)$ and $\mathscr{R}\left(\mathcal{O}_{2}+n a\right)$ $\subseteq \mathscr{R}\left(\mathcal{O}_{2}\right)$ for all $n \in \mathbb{N}$. Because of the cluster property of the vacuum every sequence of local operators $C(n a)$ converges weakly and the limit is just the vacuum expectation value of $C: \underset{n \rightarrow \infty}{\mathrm{w}-\lim } C(n a)=\varphi_{0}(C) \cdot \mathbb{1}$. Now assume that there exists a normal product state $\varphi$ for the rings $\mathscr{R}\left(\mathcal{U}_{1}\right)$ and $\mathscr{R}\left(\mathcal{O}_{2}\right)$ and take any pair of operators $A \in \mathscr{R}\left(\mathcal{O}_{1}\right)$ and $B \in \mathscr{R}\left(\mathcal{O}_{2}\right)$. Then

$$
\varphi_{0}(A B)=\lim _{n \rightarrow \infty} \varphi(A(n a) B(n a))=\lim _{n \rightarrow \infty} \varphi(A(n a)) \cdot \varphi(B(n a))=\varphi_{0}(A) \varphi_{0}(B) .
$$

But the vacuum is not a product state for $\mathscr{R}\left(\mathcal{O}_{1}\right)$ and $\mathscr{R}\left(\mathcal{O}_{2}\right)$ because it is cyclic for these rings. Thus there does not exist any normal product state for $\mathscr{R}\left(\mathcal{O}_{1}\right)$ and $\mathscr{R}\left(\mathcal{O}_{2}\right)$.

It is a remarkable fact that for both configurations of $\mathcal{O}_{1}$ and $\mathcal{O}_{2}$ the results of Roos are still valid - at least if the local rings are factors. The difficulties quoted above only occur if one insists on the product state being a normal state within a physically interesting representation.

\footnotetext{
4 This example is due to Araki.
} 


\section{The Product State for the Free Field}

In this chapter we shall show that there exists a normal product state for $\mathscr{R}\left(\mathcal{O}_{1}\right)$ and $\mathscr{R}\left(\mathcal{O}_{2}\right)$ in the case of the free neutral massive scalar field. To avoid unnecessary complications let us assume that the region $\mathcal{O}_{1}$ is the interior of the causal shadow of a compact region $\boldsymbol{O}_{1} \subset \mathbb{R}^{3}$ at time $t=0$. With other words: $\mathcal{O}_{1}$ is the "double cone" generated by $\boldsymbol{O}_{1}$ at $t=0 . \mathcal{O}_{2}$ shall be generated in the same way by a region $\boldsymbol{O}_{2} \subset \mathbb{R}^{3}$ with compact complement. Both regions shall have smooth boundaries and the distance between $\boldsymbol{O}_{1}$ and $\boldsymbol{O}_{2}$ is supposed to be greater than zero. (All other interesting configurations can be reduced to this one by first making a Poincaré transformation and then restricting to subregions of $\mathcal{O}_{1}$ and $\mathcal{O}_{2}$.)

These restrictions on the regions make it possible to use the time-slice description for the free field. We adapt our notation to that of Araki [6] and work with the following quantities: let $K$ be a direct sum of Schwartzspaces, $K=\mathscr{S}\left(\mathbb{R}^{3}\right) \oplus \mathscr{S}\left(\mathbb{R}^{3}\right)$. We shall denote the elements of $K$ by capital letters $F, G$. $K$ is the testfunction space for the field operators,

$$
B(F)=\pi(f)+\phi(g), F=f \oplus g \in K .
$$

$(\phi($.$) denotes the "field" and \pi($.$) its canonically conjugate "momentum".)$ The adjoint of $B(F)$ is

$$
B(F)^{*}=B(\Gamma F),
$$

$\Gamma$ being the antilinear operator of complex conjugation, $\Gamma F=\bar{F}$. The commutator of the field $B($.$) defines a hermitian form \gamma$ on $K$ :

$$
\gamma(F, G) \cdot \mathbb{1} \equiv B(F)^{*} B(G)-B(G) B(F)^{*}=\left(F,\left(\begin{array}{rr}
0 & -i \\
i & 0
\end{array}\right) G\right)^{5} .
$$

With this structure the *-algebra $\mathfrak{A}(K, \gamma, \Gamma)$ generated by all polynomials of the operators $B(F)$ is a "self-dual CCR algebra" in the sense of Araki [6].

Now we come to the definition of the vacuum functional $\varphi_{0}$ of $\mathfrak{A}(K, \gamma, \Gamma)$. As a quasifree state $\varphi_{0}$ is completely determined by its 2-point function

$$
S_{0}(F, G) \equiv \varphi_{0}\left(B(F)^{*} B(G)\right)=\frac{1}{2}\left(F,\left(\begin{array}{rr}
1 & -i \\
i & 1
\end{array}\right) G\right) .
$$

With this definition of the vacuum $\varphi_{0}$ the correspondence between regions in configuration space and sets in the testfunction space $K$ is as follows:

\footnotetext{
${ }^{5}$ Here $(.,$.$) denotes the scalar product in L^{2}\left(\mathbb{R}^{3}\right) \oplus L^{2}\left(\mathbb{R}^{3}\right)$. We shall also use this symbol for the scalar product in $L^{2}\left(\mathbb{R}^{3}\right)$, but we think that no confusion will arise because we shall always use capital letters for elements of $L^{2}\left(\mathbb{R}^{3}\right) \oplus L^{2}\left(\mathbb{R}^{3}\right)$ and small letters for elements of $L^{2}\left(\mathbb{R}^{3}\right)$.
} 
the "local" self-dual CCR algebra $\mathfrak{A}(K(\boldsymbol{O}), \gamma, \Gamma)$ attached to the region $\boldsymbol{O} \subset \mathbb{R}^{3}$ at time $t=0$ is generated by the field-operators $B(F)$ with testfunctions $F \in K(\boldsymbol{O})$,

$$
K(\boldsymbol{O})=\omega^{1 / 2} \mathscr{S}(\boldsymbol{O}) \oplus \omega^{-1 / 2} \mathscr{S}(\boldsymbol{O}) .
$$

(The operator $\omega$ is diagonal in momentum space: $\tilde{\omega}(\boldsymbol{p})=\left(|\boldsymbol{p}|^{2}+m^{2}\right)^{1 / 2}$, $m \neq 0$ being the mass. $\mathscr{S}(\boldsymbol{O})$ denotes the elements in $\mathscr{S}\left(\mathbb{R}^{3}\right)$ with support in $\boldsymbol{O}$.) The local rings $\mathscr{R}(\mathcal{O})(\mathcal{O}$ being the causal shadow of the region $\boldsymbol{O} \subset \mathbb{R}^{3}$ at time $t=0$ ) are generated by the spectral projections of the selfadjoint elements of the algebras $\mathfrak{A}(K(\boldsymbol{O}), \gamma, \Gamma)$ in the vacuum representation.

Let us now briefly sketch how we shall proceed in order to show that there exist normal product states for $\mathscr{R}\left(\mathcal{O}_{1}\right)$ and $\mathscr{R}\left(\mathcal{O}_{2}\right)$ : from the considerations in Chapter II we know that one has only to check whether there exists a normal product state for $\mathscr{R}\left(\mathcal{O}_{1}\right)$ and $\mathscr{R}\left(\mathcal{O}_{2}\right)$ which coincides with the vacuum $\varphi_{0}$ on each algebra separately. Therefore we start with a positive functional $\varphi_{P}$ on $\mathscr{R}\left(\mathcal{O}_{1}\right) \vee \mathscr{R}\left(\mathcal{O}_{2}\right)$ (the algebra of all finite sums $\sum A_{n} B_{n}, A_{n} \in \mathscr{R}\left(\mathcal{O}_{1}\right)$ and $\left.B_{n} \in \mathscr{R}\left(\mathcal{O}_{2}\right)\right)$ which is defined by

$\varphi_{p}\left(\Sigma A_{n} B_{n}\right)=\Sigma \varphi_{0}\left(A_{n}\right) \varphi_{0}\left(B_{n}\right)$ for $A_{n} \in \mathscr{R}\left(\mathcal{O}_{1}\right)$ and $B_{n} \in \mathscr{R}\left(\mathcal{O}_{2}\right)$.

Clearly $\varphi_{p}$ induces a representation of the self-dual CCR algebra $\mathfrak{A}\left(K\left(\boldsymbol{O}_{1} \cup \boldsymbol{O}_{2}\right), \gamma, \Gamma\right)$. Since the vacuum is a quasifree state of the CCR it follows from the definition that $\varphi_{p}$ is a quasifree state of the CCR too. Hence we have to compare two quasifree representations of the CCR which are primary (as we shall see). Now Araki [7] has given a criterion which tells us when two such representations are equivalent. So we have only to check his conditions in order to verify that $\varphi_{p}$ can be extended to a normal product state for $\mathscr{R}\left(\mathcal{O}_{1}\right)$ and $\mathscr{R}\left(\mathcal{O}_{2}\right)$.

To carry through this program we need some more definitions. We introduce on $K(\boldsymbol{O}), \boldsymbol{O}=\boldsymbol{O}_{1} \cup \boldsymbol{O}_{2}$ a scalar product $(.,)_{0}$ :

$$
(F, G)_{0} \equiv S_{0}(F, G)+S_{0}(\Gamma G, \Gamma F)=(F, G) ;\|F\|_{0}^{2}=(F, F)_{0} .
$$

The completion of $K(\boldsymbol{O})$ with respect to $\|.\|_{0}$ is denoted by $K_{0}(\boldsymbol{O}) ; K_{0}(\boldsymbol{O})$ is a closed subspace of $L^{2}\left(\mathbb{R}^{3}\right) \oplus L^{2}\left(\mathbb{R}^{3}\right)$. On $K_{0}(\boldsymbol{O})$ we can represent the positive form $S_{0}(.,$.$) (see relation (3)) by a positive operator S_{0}$ which is bounded by 1 :

$$
S_{0}=\frac{1}{2} E\left(\begin{array}{rr}
1 & -i \\
i & 1
\end{array}\right) E ;\left(F, S_{0} G\right)_{0}=S_{0}(F, G) \quad \text { for } \quad F, G \in K_{0}(\boldsymbol{O}) .
$$

(Here $E$ denotes the orthogonal projection in $L^{2}\left(\mathbb{R}^{3}\right) \oplus L^{2}\left(\mathbb{R}^{3}\right)$ onto $K_{0}(\boldsymbol{O})$.) The restriction of $\varphi_{0}$ to $\mathfrak{A}(K(\boldsymbol{O}), \gamma, \Gamma)$ is then completely determined by $S_{0}$. 
Next we consider the quasifree state $\varphi_{p}$ introduced by relation (4). Because of the non-zero distance of $\boldsymbol{O}_{1}$ and $\boldsymbol{O}_{2}$, every element $F \in K(\boldsymbol{O})$ can be decomposed uniquely into a sum of an element $F_{1} \in K\left(\boldsymbol{O}_{1}\right)$ and an element $F_{2} \in K\left(\boldsymbol{O}_{2}\right)$. From this and relation (4) one gets for the 2-point function:

$$
S_{p}(F, G) \equiv \varphi_{p}\left(B(F)^{*} B(G)\right)=S_{0}\left(F_{1}, G_{1}\right)+S_{0}\left(F_{2}, G_{2}\right) .
$$

Now we proceed as above: we introduce on $K(\boldsymbol{O})$ another scalar product $(., .)_{p}$

$$
(F, G)_{p} \equiv S_{p}(F, G)+S_{p}(\Gamma G, \Gamma F)=\left(F_{1}, G_{1}\right)_{0}+\left(F_{2}, G_{2}\right)_{0} ;\|F\|_{p}^{2}=(F, F)_{p} .
$$

The completion of $K(\boldsymbol{O})$ with respect to $\|\cdot\|_{p}$ is denoted by $K_{p}(\boldsymbol{O})$. From (8) and the definition of the decomposition $F=F_{1}+F_{2}$ it follows immediately that there exist two mutually orthogonal projections $\Theta_{1}$ and $\Theta_{2}$ on $K_{p}(\boldsymbol{O})$ which satisfy $\Theta_{1} F=F_{1}$ and $\Theta_{2} F=F_{2}$ for all $F \in K(\boldsymbol{O})$. Thus $K_{p}(\boldsymbol{O})$ can be considered as a direct sum of $K_{0}\left(\boldsymbol{O}_{1}\right)$ and $K_{0}\left(\boldsymbol{O}_{2}\right)$.

With the help of $\Theta_{1}$ and $\Theta_{2}$ we can represent the positive form $S_{p}(.,$.$) on K_{p}(\boldsymbol{O})$ by a positive operator $S_{p}$ which is bounded by 1 :

$$
\begin{gathered}
S_{p}=\frac{1}{2} E_{1}\left(\begin{array}{rr}
1 & -i \\
i & 1
\end{array}\right) E_{1} \Theta_{1}+\frac{1}{2} E_{2}\left(\begin{array}{rr}
1 & -i \\
i & 1
\end{array}\right) E_{2} \Theta_{2} ; \\
\left(F, S_{p} G\right)_{p}=S_{p}(F, G) \text { for } F, G \in K_{p}(O) .
\end{gathered}
$$

(Here $E_{1}, E_{2}$ are the orthogonal projections in $L^{2}\left(\mathbb{R}^{3}\right) \oplus L^{2}\left(\mathbb{R}^{3}\right)$ onto $K_{0}\left(\boldsymbol{O}_{1}\right)$ and $K_{0}\left(\boldsymbol{O}_{2}\right)$ respectively.) Now we are prepared to formulate the criterion given by Araki [7; Lemma 6.2 and 6.5].

Criterion. The representations $\pi_{0}$ and $\pi_{p}$ of $\mathscr{R}\left(\mathcal{O}_{1}\right) \vee \mathscr{R}\left(\mathcal{O}_{2}\right)$ induced by $\varphi_{0}$ and $\varphi_{p}$ respectively are unitarily equivalent if the following conditions hold:

i) $S_{0}$ and $S_{p}$ do not have the eigenvalue $\frac{1}{2}$ in $K_{0}(\boldsymbol{O})$ and $K_{p}(\boldsymbol{O})$ respectively.

ii) The norms $\|\cdot\|_{0}$ and $\|\cdot\|_{p}$ are equivalent on $K(\boldsymbol{O})$.

iii) The operators $\left(S_{0}-S_{p}\right)\left(\mathbb{1}-2 S_{0}\right)^{-1}$ and $\sqrt{S_{0}\left(\mathbb{1}-S_{0}\right)}-\sqrt{S_{p}\left(\mathbb{1}-S_{p}\right)}$ are of Hilbert-Schmidt class in $K_{0}(\boldsymbol{O})$. (Here we have identified $K_{0}(\boldsymbol{O})$ and $K_{p}(\boldsymbol{O})$ using Condition ii). The positive square root of $S_{0}\left(\mathbb{1}-S_{0}\right)$ is relative to $(., .)_{0}$ and that of $S_{p}\left(\mathbb{1}-S_{p}\right)$ is relative to $\left.(., .)_{p}.\right)^{6}$

iv) $S_{0}$ and $S_{p}$ do not have the eigenvalue 0 in $K_{0}(\boldsymbol{O})$ and $K_{p}(\boldsymbol{O})$ respectively.

For a better understanding of this criterion let us illustrate which properties are imposed by these conditions on the representations $\pi_{0}$

${ }^{6}$ Our Condition iii) differs from the condition Araki has given. We shall show in Appendix A that both conditions are equivalent. 
and $\pi_{p}$ : Condition i) is equivalent to the requirement that both representations are primary. It is well known that this is the case for the representation $\pi_{0}$ [8] and it follows from the tensor product structure of the representation induced by $\varphi_{p}$ that $\pi_{p}$ is primary too. If in addition the Conditions ii) and iii) are satisfied then the two representations are quasiequivalent. This would be already enough to prove that $\pi_{0}$ and $\pi_{p}$ are unitarily equivalent because one knows that the local rings are Type III [8]. Hence it would actually not be necessary to check Condition iv) which says that $\varphi_{0}$ and $\varphi_{p}$ must be separating states. Yet for the reader's convenience we shall prove explicitly that all the conditions given by Araki are fulfilled in our special case. Let us start with the first one:

Lemma 3.1. $S_{0}$ and $S_{p}$ do not have the eigenvalue $\frac{1}{2}$ in $K_{0}(\boldsymbol{O})$ and $K_{p}(\boldsymbol{O})$ respectively.

Proof. i) Let $E_{\pi}$ and $E_{\phi}$ be the projections in $L^{2}\left(\mathbb{R}^{3}\right)$ onto the closed subspaces $\left[\omega^{1 / 2} \mathscr{S}(\boldsymbol{O})\right]$ and $\left[\omega^{-1 / 2} \mathscr{S}(\boldsymbol{O})\right]$ respectively. Then the projection $E$ in $L^{2}\left(\mathbb{R}^{3}\right) \oplus L^{2}\left(\mathbb{R}^{3}\right)$ onto $K_{0}(\boldsymbol{O})$ can be expressed in the following way:

$$
E=\left(\begin{array}{cc}
E_{\pi} & 0 \\
0 & E_{\phi}
\end{array}\right) \text { and therefore } S_{0}-\frac{1}{2} E=\frac{1}{2}\left(\begin{array}{cc}
0 & -i E_{\pi} E_{\phi} \\
i E_{\phi} E_{\pi} & 0
\end{array}\right)
$$

Thus $\left(S_{0}-\frac{\mathbb{1}}{2}\right) F=0$ in $K_{0}(\boldsymbol{O})$ implies that $E_{\phi} E_{\pi} f=E_{\pi} E_{\phi} g=0$ for certain elements $f \in\left[\omega^{1 / 2} \mathscr{S}(\boldsymbol{O})\right]$ and $g \in\left[\omega^{-1 / 2} \mathscr{S}(\boldsymbol{O})\right]$. We want to show that $f=g=0$.

Take any element $h \in L^{2}\left(\mathbb{R}^{3}\right) . h$ can be decomposed into a sum $h=h_{1}+h_{2}$ with $h_{1} \in L^{2}(\boldsymbol{O})$ and $h_{2} \in L^{2}\left(\mathbb{R}^{3}-\boldsymbol{O}\right)$. Since we can find for each $f \in\left[\omega^{1 / 2} \mathscr{S}(\boldsymbol{O})\right]$ a sequence $\varphi_{n} \in \mathscr{S}(\boldsymbol{O})$ such that $f=\operatorname{s}-\lim \omega^{1 / 2} \varphi_{n}$, we get

$$
\begin{aligned}
\left(\omega^{-1 / 2} h, f\right) & =\lim \left(\omega^{-1 / 2} h, \omega^{1 / 2} \varphi_{n}\right)=\lim \left(\omega^{-1 / 2} h_{1}, \omega^{1,2} \varphi_{n}\right) \\
& =\left(\omega^{-1 / 2} h_{1}, E_{\phi} E_{\pi} f\right)=0 .
\end{aligned}
$$

From this it follows that $\omega^{-1 / 2} f=0$ and therefore $f$ must be zero.

In order to prove that $g=0$ we have to be a little bit more careful. Let $\mathscr{D}_{\omega^{1 / 2}}$ be the domain of $\omega^{1 / 2}$. The elements $h \in L^{2}\left(\mathbb{R}^{3}\right)$ which can be expressed as $h=h_{1}+h_{2}$ with $h_{1} \in L^{2}(\boldsymbol{O}) \cap \mathscr{D}_{\omega^{1 / 2}}$ and $h_{2} \in L^{2}\left(\mathbb{R}^{3}-\boldsymbol{O}\right) \cap \mathscr{D}_{\omega^{1 / 2}}$ are a core for $\omega^{1 / 2}$ because the boundary of $\boldsymbol{O}$ is smooth [8]. Now we can proceed as above: every $g \in\left[\omega^{-1 / 2} \mathscr{S}(\boldsymbol{O})\right]$ is a strong limit $g=\lim \omega^{-1 / 2} \psi_{n}$ with $\psi_{n} \in \mathscr{S}(\boldsymbol{O})$. Take any $h$ from the set described above. Then

$$
\begin{aligned}
\left(\omega^{1 / 2} h, g\right) & =\lim \left(\omega^{1 / 2} h, \omega^{-1 / 2} \psi_{n}\right)=\lim \left(\omega^{1 / 2} h_{1}, \omega^{-1 / 2} \psi_{n}\right) \\
& =\left(\omega^{1 / 2} h_{1}, E_{\pi} E_{\phi} g\right)=0
\end{aligned}
$$


From this it follows that $g \in \mathscr{D}_{\omega^{1 / 2}}$ and $\omega^{1 / 2} g=0$, hence $g=0$. Thus we have proved that $S_{0}$ does not have the eigenvalue $\frac{1}{2}$ in $K_{0}(\boldsymbol{O})$.

ii) Let us now assume that $\left(S_{p}-\frac{\mathbb{1}}{2}\right) F=0$ for some $F \in K_{p}(\boldsymbol{O})$. From relation (8) and (9) it then follows that

and

$$
E_{1}\left(\begin{array}{rr}
0 & -i \\
i & 0
\end{array}\right) E_{1} \cdot \Theta_{1} F=0 \quad \text { in } \quad K_{0}\left(\boldsymbol{O}_{1}\right)
$$

$$
E_{2}\left(\begin{array}{rr}
0 & -i \\
i & 0
\end{array}\right) E_{2} \cdot \Theta_{2} F=0 \quad \text { in } \quad K_{0}\left(\boldsymbol{O}_{2}\right)
$$

Applying the same methods as in the preceding section, one can conclude that $\Theta_{1} F=0$ in $K_{0}\left(\boldsymbol{O}_{1}\right)$ and $\Theta_{2} F=0$ in $K_{0}\left(\boldsymbol{O}_{2}\right)$; from this one immediately gets the desired result: $F=0$ in $K_{p}(\boldsymbol{O})$.

In the next step we shall prove that the norms $\|\cdot\|_{0}$ and $\|\cdot\|_{p}$ are equivalent. Actually if $\boldsymbol{O}_{1}$ and $\boldsymbol{O}_{2}$ were sufficiently far apart, this would follow from the cluster property of the vacuum. Since we want to prove the statement for arbitrary positive distances between the regions, we proceed as follows:

Lemma 3.2. The norms $\|\cdot\|_{0}$ and $\|\cdot\|_{p}$ on $K(\boldsymbol{O})$ are equivalent.

Proof. To begin with we shall show that there exists a fixed positive number $\varepsilon<1$ such that

$\left|\left(f, \omega^{-1} g\right)\right|^{2} \leqq \varepsilon^{2}\left(f, \omega^{-1} f\right)\left(g, \omega^{-1} g\right) \quad$ and $\quad|(f, \omega g)|^{2} \leqq \varepsilon^{2}(f, \omega f)(g, \omega g)$ for all $f \in \mathscr{S}\left(\boldsymbol{O}_{1}\right)$ and $g \in \mathscr{S}\left(\boldsymbol{O}_{2}\right)$. Let $d$ be the distance between $\boldsymbol{O}_{1}$ and $\boldsymbol{O}_{2}$ and consider the function

$$
s(\boldsymbol{p})=\int_{|\boldsymbol{x}| \leqq \frac{d}{2}} d^{3} x \frac{1}{|\boldsymbol{x}|^{2}} e^{-i(\boldsymbol{p} \boldsymbol{x})} .
$$

It is easy to verify that $\infty>c_{1} \geqq\left(|\boldsymbol{p}|^{2}+m^{2}\right)^{1 / 2} s(\boldsymbol{p}) \geqq c_{2}>0$. Thus one can find a number $\delta$ and a positive number $\varepsilon<1$ such that

and

$$
\left|\left(|\boldsymbol{p}|^{2}+m^{2}\right)^{-1 / 2}-\delta \cdot s(\boldsymbol{p})\right| \leqq \varepsilon \cdot\left(|\boldsymbol{p}|^{2}+m^{2}\right)^{-1 / 2}
$$

$$
\left|\left(|\boldsymbol{p}|^{2}+m^{2}\right)^{1 / 2}-\delta \cdot\left(|\boldsymbol{p}|^{2}+m^{2}\right) s(\boldsymbol{p})\right| \leqq \varepsilon \cdot\left(|\boldsymbol{p}|^{2}+m^{2}\right)^{1 / 2}
$$

Let $\omega^{\#}, \omega_{\text {\# }}$ be two operators which are diagonal in momentum space,

$$
\omega^{\#}(\boldsymbol{p})=\delta \cdot s(\boldsymbol{p}) \text { and } \omega_{\#}(\boldsymbol{p})=\delta \cdot\left(|\boldsymbol{p}|^{2}+m^{2}\right) s(\boldsymbol{p}) .
$$

Take any element $g \in \mathscr{S}\left(\boldsymbol{O}_{2}\right)$. It follows immediately from the definition of $s(\boldsymbol{p})$ that $\omega^{\#} g$ and $\omega_{\#} g$ are elements of $\mathscr{S}\left(\mathbb{R}^{3}\right)$ and that the supports 
of $\omega^{\#} g$ and $\omega_{\#} g$ in $\boldsymbol{x}$-space are separated from $\boldsymbol{O}_{1}$. Thus one gets

$$
\left(f, \omega^{\#} g\right)=\left(f, \omega_{\#} g\right)=0 \text { for all } f \in \mathscr{S}\left(\boldsymbol{O}_{1}\right) \text { and } g \in \mathscr{S}\left(\boldsymbol{O}_{2}\right) \text {. }
$$

From this it follows

$$
\begin{aligned}
& \left|\left(f, \omega^{-1} g\right)\right|=\left|\left(f,\left[\omega^{-1}-\omega^{\#}\right] g\right)\right| \leqq \int d^{3} p\left|\left(|\boldsymbol{p}|^{2}+m^{2}\right)^{-1 / 2}-\delta \cdot s(\boldsymbol{p})\right| \cdot|\tilde{f}(\boldsymbol{p})| \\
& \cdot|\tilde{g}(\boldsymbol{p})| \leqq \varepsilon \cdot \int d^{3} p\left(|\boldsymbol{p}|^{2}+m^{2}\right)^{-1 / 2} \cdot|\tilde{f}(\boldsymbol{p})| \cdot|\tilde{g}(\boldsymbol{p})| \leqq \varepsilon\left(f, \omega^{-1} f\right)^{1 / 2}\left(g, \omega^{-1} g\right)^{1 / 2}
\end{aligned}
$$

and in the same way $|(f, \omega g)| \leqq \varepsilon(f, \omega f)^{1 / 2}(g, \omega g)^{1 / 2}$. Now it is obvious that

and

$$
\begin{gathered}
(1+\varepsilon)^{-1} \cdot([f+g], \omega[f+g]) \leqq(f, \omega f)+(g, \omega g) \\
\leqq(1-\varepsilon)^{-1} \cdot([f+g], \omega[f+g])
\end{gathered}
$$

$$
\begin{gathered}
(1+\varepsilon)^{-1} \cdot\left([f+g], \omega^{-1}[f+g]\right) \leqq\left(f, \omega^{-1} f\right)+\left(g, \omega^{-1} g\right) \\
\leqq(1-\varepsilon)^{-1} \cdot\left([f+g], \omega^{-1}[f+g]\right)
\end{gathered}
$$

for arbitrary $f \in \mathscr{S}\left(\boldsymbol{O}_{1}\right)$ and $g \in \mathscr{S}\left(\boldsymbol{O}_{2}\right)$. Recalling the definition of $\|\cdot\|_{0}$ and $\|\cdot\|_{p}$ it is trivial to conclude from these equations that

$$
(1+\varepsilon)^{-1 / 2}\|F\|_{0} \leqq\|F\|_{p} \leqq(1-\varepsilon)^{-1 / 2}\|F\|_{0} \text { for all } F \in K(\boldsymbol{O}) .
$$

Now we come to that condition in Arakis criterion which looks most complicated. It will turn out that the operator $E_{1} E_{2}$ is of trace class in $L^{2}\left(\mathbb{R}^{3}\right) \oplus L^{2}\left(\mathbb{R}^{3}\right)$ and this allows us to verify Condition iii).

Lemma 3.3. The operators $\left(S_{0}-S_{p}\right)\left(\mathbb{1}-2 S_{0}\right)^{-1}$ and $\sqrt{S_{0}\left(\mathbb{1}-S_{0}\right)}$ $-\sqrt{S_{p}\left(\mathbb{1}-S_{p}\right)}$ are of Hilbert-Schmidt class in $K_{0}(\boldsymbol{O})$.

Proof. Starting from the relations (6) and (9) a straightforward calculation shows that

i) $\left(S_{p}-S_{0}\right)\left(\mathbb{1}-2 S_{0}\right)^{-1}=E-E_{1}-E_{2}$

and

ii) $\sqrt{S_{0}\left(\mathbb{1}-S_{0}\right)}-\sqrt{S_{p}\left(\mathbb{1}-S_{p}\right)}=\{E T(\mathbb{1}-E) T E\}^{1 / 2}$ $-\left\{E_{1} T\left(\mathbb{1}-E_{1}\right) T E_{1}\right\}^{1 / 2} \Theta_{1}-\left\{E_{2} T\left(\mathbb{1}-E_{2}\right) T E_{2}\right\}^{1 / 2} \Theta_{2}$.

(Here we have introduced the operator $T=\left(\begin{array}{ll}0 & 1 \\ 1 & 0\end{array}\right)$. The symbol $\{.\}^{1 / 2}$ says that the positive square root has to be taken in $L^{2}\left(\mathbb{R}^{3}\right) \oplus L^{2}\left(\mathbb{R}^{3}\right)$.)

i) It follows from the definition of $\Theta_{1}$ and $\Theta_{2}$ that $\Theta_{1}+\Theta_{2}=\mathbb{1}$ in $K_{0}(\boldsymbol{O})$. Thus one gets

$$
\begin{aligned}
E-E_{1}-E_{2} & =\left(E-E_{1}-E_{2}\right)\left(\Theta_{1}+\Theta_{2}\right)=\left(E-E_{1}-E_{2}\right)\left(E_{1} \Theta_{1}+E_{2} \Theta_{2}\right) \\
& =-E_{2} E_{1} \Theta_{1}-E_{1} E_{2} \Theta_{2} .
\end{aligned}
$$


Hence if $E_{1} E_{2}$ is of trace class in $K_{0}(\boldsymbol{O}),\left(S_{p}-S_{0}\right)\left(\mathbb{1}-2 S_{0}\right)^{-1}$ is of trace class too and thus a fortiori of Hilbert-Schmidt class.

ii) Let us next prove that the operator

$$
\begin{aligned}
\{E T(\mathbb{1}-E) T E\}^{1 / 2} & -\left\{E_{1} T\left(\mathbb{1}-E_{1}\right) T E_{1}\right\}^{1 / 2} \Theta_{1} \\
& -\left\{E_{2} T\left(\mathbb{1}-E_{2}\right) T E_{2}\right\}^{1 / 2} \Theta_{2}
\end{aligned}
$$

is of Hilbert-Schmidt class in $K_{0}(\boldsymbol{O})$ if $E_{1} E_{2}$ is of trace class. It follows from the definition of $\Theta_{1}$ and $\Theta_{2}$ that

$\Theta_{1}=E_{1} \Theta_{1}=E_{1}-E_{1} \Theta_{2}=E_{1}-E_{1} E_{2} \cdot \Theta_{2}$ and $\Theta_{2}=E_{2}-E_{2} E_{1} \cdot \Theta_{1}$

and therefore we can replace $\Theta_{1}$ and $\Theta_{2}$ in the expression (a) by $E_{1}$ and $E_{2}$ respectively, the difference being of trace class. Therefore we are left with the question of whether

$$
\{E T(\mathbb{1}-E) T E\}^{1 / 2}-\left\{E_{1} T\left(\mathbb{1}-E_{1}\right) T E_{1}\right\}^{1 / 2}-\left\{E_{2} T\left(\mathbb{1}-E_{2}\right) T E_{2}\right\}^{1 / 2}
$$

is of Hilbert-Schmidt class. In Appendix B it is shown that the operator $\left\{X^{2}+Y\right\}^{1 / 2}-X \quad\left(\infty>X \geqq 0\right.$ and $\left.X^{2}+Y \geqq 0\right)$ is of Hilbert-Schmidt class if $Y$ is of trace class. If one puts $X=\left\{E_{1} T\left(\mathbb{1}-E_{1}\right) T E_{1}\right\}^{1 / 2}$ $+\left\{E_{2} T\left(\mathbb{1}-E_{2}\right) T E_{2}\right\}^{1 / 2}$ and $X^{2}+Y=E T(\mathbb{1}-E) T E$ one has only to verify that

(b) $\quad E T(\mathbb{1}-E) T E-E_{1} T\left(\mathbb{1}-E_{1}\right) T E_{1}-E_{2} T\left(\mathbb{1}-E_{2}\right) T E_{2}$

is of trace class. (Here we have omitted the "mixed terms" $\left\{E_{1} T\left(\mathbb{1}-E_{1}\right) T E_{1}\right\}^{1 / 2} \cdot\left\{E_{2} T\left(\mathbb{1}-E_{2}\right) T E_{2}\right\}^{1 / 2}$ etc., again using the fact that $E_{1} E_{2}$ is of trace class.) Now we know from our preceding considerations that one can replace in expression (b) the operator $E$ by $E_{1}+E_{2}$, the error being of trace class. It is furthermore easy to show that $E_{1} T E_{2}=E_{2} T E_{1}=0$ and $T \cdot T=\mathbb{1}$. Bearing this in mind, it is almost evident that the operator given by expression (b) has the desired properties.

iii) Finally we have to show that the operator $E_{1} E_{2}$ is really of trace class. For this purpose we express $E_{1}$ and $E_{2}$ as follows:

$$
E_{1}=\left(\begin{array}{cc}
E_{\pi}^{(1)} & 0 \\
0 & E_{\phi}^{(1)}
\end{array}\right) \text { and } E_{2}=\left(\begin{array}{cc}
E_{\pi}^{(2)} & 0 \\
0 & E_{\phi}^{(2)}
\end{array}\right) \text {, }
$$

$E_{\pi}^{(k)}, E_{\phi}^{(k)} k=1,2$ being the projections onto the closed subspaces $\left[\omega^{1 / 2} \mathscr{S}\left(\boldsymbol{O}_{k}\right)\right]$ and $\left[\omega^{-1 / 2} \mathscr{S}\left(\boldsymbol{O}_{k}\right)\right]$ of $L^{2}\left(\mathbb{R}^{3}\right)$ respectively. Thus it suffices to show that $E_{\pi}^{(1)} E_{\pi}^{(2)}$ and $E_{\phi}^{(1)} E_{\phi}^{(2)}$ are of trace class in $L^{2}\left(\mathbb{R}^{3}\right)$.

Now let $\vartheta_{1}$ be an element of $\mathscr{S}\left(\mathbb{R}^{3}\right)$ and $\vartheta_{2}$ be an element of $\mathcal{O}_{M}\left(\mathbb{R}^{3}\right)$ (the space of slowly increasing $\mathscr{C}^{\infty}$ functions) such that the distance between the supports of $\vartheta_{1}$ and $\vartheta_{2}$ is non-zero and

$$
\vartheta_{1}(x)=1 \quad \text { for } \quad \boldsymbol{x} \in \boldsymbol{O}_{1}, \quad \vartheta_{2}(x)=1 \text { for } \boldsymbol{x} \in \boldsymbol{O}_{2} .
$$


(Such functions exist since the region $\boldsymbol{O}_{1}$ is compact and the distance between $\boldsymbol{O}_{1}$ and $\boldsymbol{O}_{2}$ is greater than zero.) Let $\Delta_{1}, \Delta_{2}$ be the operators of multiplication by $\vartheta_{1}(x)$ and $\vartheta_{2}(x)$ in $x$-space respectively. Then it is easy to verify that the operators

$$
\omega^{-1 / 2} \Delta_{1} \omega^{1 / 2} \text { and } \omega^{1 / 2} \Delta_{2} \omega^{-1 / 2}=\mathbb{1}+\omega^{1 / 2}\left(\Delta_{2}-\mathbb{1}\right) \omega^{-1 / 2}
$$

are bounded in $L^{2}\left(\mathbb{R}^{3}\right)$ if one notes that $\left(\vartheta_{2}-1\right)$ is an element of $\mathscr{C}^{\infty}$ with support in the (compact) complement of $\boldsymbol{O}_{2}$. From the definition of $E_{\pi}^{(1)}$ and $E_{\pi}^{(2)}$ it follows furthermore that

hence

$$
E_{\pi}^{(1)} \omega^{-1 / 2} \Delta_{1} \omega^{1 / 2}=E_{\pi}^{(1)} \text { and } \omega^{1 / 2} \Delta_{2} \omega^{-1 / 2} E_{\pi}^{(2)}=E_{\pi}^{(2)}
$$

$$
E_{\pi}^{(1)} E_{\pi}^{(2)}=E_{\pi}^{(1)} \omega^{-1 / 2} \Delta_{1} \omega \Delta_{2} \omega^{-1 / 2} E_{\pi}^{(2)} .
$$

Thus it suffices to show that $\omega^{-1 / 2} \Delta_{1} \omega \Delta_{2} \omega^{-1 / 2}$ is of trace class in $L^{2}\left(\mathbb{R}^{3}\right)$. Now the function

$$
\omega(\boldsymbol{z})=(2 \pi)^{-3} \int d^{3} p\left(|\boldsymbol{p}|^{2}+m^{2}\right)^{1 / 2} e^{i(\boldsymbol{p} z)}, \quad z \neq 0
$$

is arbitrarily often differentiable for $z \neq 0$ and it decreases (with all its derivatives) faster than any inverse power of $|z|$ if $|z| \rightarrow \infty$. Therefore the kernel of $\Delta_{1} \omega \Delta_{2}$ in $\boldsymbol{x}$-space,

$$
(\boldsymbol{x})\left(\Delta_{1} \omega \Delta_{2}\right)(\boldsymbol{y})=\vartheta_{1}(\boldsymbol{x}) \omega(\boldsymbol{x}-\boldsymbol{y}) \vartheta_{2}(\boldsymbol{y})
$$

is an element of $\mathscr{S}\left(\mathbb{R}^{6}\right)$. Passing to momentum space, it is obvious, that the kernel of $\omega^{-1 / 2} \Delta_{1} \omega \Delta_{2} \omega^{-1 / 2}$ is also an element of $\mathscr{S}\left(\mathbb{R}^{6}\right)$ and from this fact it follows that $\omega^{-1 / 2} \Delta_{1} \omega \Delta_{2} \omega^{-1 / 2}$ is a trace class operator in $L^{2}\left(\mathbb{R}^{3}\right)$. Thus we have proved that $E_{\pi}^{(1)} E_{\pi}^{(2)}$ is of trace class in $L^{2}\left(\mathbb{R}^{3}\right)$. If one applies the same methods to $E_{\phi}^{(1)} E_{\phi}^{(2)}$ one can conclude that this operator has the same property.

It remains to prove that the last condition of the criterion is also satisfied.

Lemma 3.4. $S_{0}$ and $S_{p}$ do not have the eigenvalue 0 in $K_{0}(\boldsymbol{O})$ and $K_{p}(\boldsymbol{O})$ respectively.

Proof. i) Let $F=f \oplus g$ be an element of $K_{0}(\boldsymbol{O})$ such that

$$
S_{0} F=0, \quad \text { hence } E\left(\begin{array}{rr}
1 & i \\
-i & 1
\end{array}\right) \cdot S_{0} F=0
$$

From this one gets for the components of $F$ after a simple calculation

$$
E_{\pi} f=E_{\phi} f=f \quad \text { and } \quad E_{\pi} g=E_{\phi} g=g .
$$

It is well known that for arbitrary open regions $\hat{\boldsymbol{O}} \subset \mathbb{R}^{3}$ the linear span of $\mathscr{S}(\hat{\boldsymbol{O}})$ and $\omega \mathscr{S}(\hat{\boldsymbol{O}})$ is dense in $L^{2}\left(\mathbb{R}^{3}\right)$. (This follows immediately from the fact that there do not exist positive energy solutions of the Klein-Gordon 
equation which vanish in open regions of $\mathbb{R}^{4}$.) Now take any $h \in \mathscr{S}\left(\mathbb{R}^{3}-\boldsymbol{O}\right)$. Since $f=\mathrm{s}-\lim \omega^{1 / 2} \varphi_{n}=\mathrm{s}-\lim \omega^{-1 / 2} \psi_{n}$ for suitable sequences $\varphi_{n}, \psi_{n} \in \mathscr{S}(\boldsymbol{O})$ one can conclude

$$
\left(h, \omega^{-1 / 2} f\right)=\left(\omega h, \omega^{-1 / 2} f\right)=0 .
$$

Thus we have proved $\omega^{-1 / 2} f=0$ and from this follows $f=0$. With the same methods one shows $g=0$, hence $F=0$.

ii) Let us assume next that there exists a $F \in K_{p}(\boldsymbol{O})$ such that $S_{p} F=0$. From this one gets the relations

and

$$
E_{1}\left(\begin{array}{rr}
1 & -i \\
i & 1
\end{array}\right) E_{1} \cdot \Theta_{1} F=0 \quad \text { in } \quad K_{0}\left(\boldsymbol{O}_{1}\right)
$$

$$
E_{2}\left(\begin{array}{rr}
1 & -i \\
i & 1
\end{array}\right) E_{2} \cdot \Theta_{2} F=0 \quad \text { in } \quad K_{0}\left(\boldsymbol{O}_{2}\right)
$$

If one performs the same calculations as above one gets $\Theta_{1} F=0$ in $K_{0}\left(\boldsymbol{O}_{1}\right)$ and $\Theta_{2} F=0$ in $K_{0}\left(\boldsymbol{O}_{2}\right)$, hence $F=0$ in $K_{p}(\boldsymbol{O})$.

Now we are finished: since all the conditions are satisfied it follows from the criterion that the representations $\pi_{0}$ and $\pi_{p}$ are unitarily equivalent. Thus $\varphi_{p}$ can be extended to a normal state of $\mathscr{R}\left(\mathscr{R}\left(\mathcal{O}_{1}\right), \mathscr{R}\left(\mathcal{O}_{2}\right)\right)$. Lemma 2.1 guarantees that this extension of $\varphi_{p}$ can be represented by a vector $\eta \in \mathscr{H}$. Hence there exists a normal product state for $\mathscr{R}\left(\mathcal{O}_{1}\right)$ and $\mathscr{R}\left(\mathrm{O}_{2}\right)$.

Theorem 3.5. Let $\mathcal{O}_{1}$ be a bounded region such that $\mathcal{O}_{1}+\mathcal{N}$ is spacelike separated from the region $\mathcal{O}_{2}$. Let $\mathscr{R}\left(\mathcal{O}_{1}\right)$ and $\mathscr{R}\left(\mathcal{O}_{2}\right)$ be the local rings associated with these regions in the vacuum representation of the free neutral massive scalar field. Then there exists a vector $\eta \in \mathscr{H}$ such that

$$
\varphi_{\eta}(A B)=\varphi_{0}(A) \cdot \varphi_{0}(B) \text { for all } A \in \mathscr{R}\left(\mathcal{O}_{1}\right) \text { and } B \in \mathscr{R}\left(\mathcal{O}_{2}\right) \text {. }
$$

\section{A Necessary and Sufficient Condition}

The existence of normal product states could be established in the free field case. Since our methods of proof have been very much adapted to the special features of this model it is opportune to say something about the general situation.

If one wants to investigate whether there exist normal product states in the vacuum representation of an algebra of observables one should start with the state $\varphi_{p}$ of $\mathscr{R}\left(\mathcal{O}_{1}\right) \vee \mathscr{R}\left(\mathcal{O}_{2}\right)$ which is defined by

$$
\varphi_{p}\left(\sum A_{n} B_{n}\right)=\Sigma \varphi_{0}\left(A_{n}\right) \varphi_{0}\left(B_{n}\right) \text { for all } A_{n} \in \mathscr{R}\left(\mathcal{O}_{1}\right), B_{n} \in \mathscr{R}\left(\mathcal{O}_{2}\right) \text {. }
$$


One knows from Theorem 2.2, that $\varphi_{p}$ is a vector state if there exists any normal product state for these algebras. In this case the norm distance of $\varphi_{0}$ and $\varphi_{p}$ with respect to $\mathscr{R}\left(\mathcal{O}_{1}\right) \vee \mathscr{R}\left(\mathcal{O}_{2}\right)$ would be less than 2 since $\varphi_{0}$ is separating for $\left\{\mathscr{R}\left(\mathcal{O}_{1}\right) \vee \mathscr{R}\left(\mathcal{O}_{2}\right)\right\}^{\prime \prime}$,

$$
\left\|\varphi_{0}-\varphi_{p}\right\|_{\mathscr{R}\left(\mathbb{O}_{1}\right) \vee \mathscr{R}\left(\mathbb{O}_{2}\right)}<2 .
$$

On the other hand this is a sufficient condition for the existence of normal product states since it implies that the representations $\pi_{0}$ and $\pi_{p}$ are not disjoint [9]. To simplify the discussion let us assume that the local rings $\mathscr{R}\left(\mathcal{O}_{1}\right)$ and $\mathscr{R}\left(\mathcal{O}_{2}\right)$ are factors. (This is a reasonable postulate in quantum field theory.) Then $\pi_{p}\left(\mathscr{R}\left(\mathcal{O}_{1}\right) \vee \mathscr{R}\left(\mathcal{O}_{2}\right)\right)^{\prime \prime} \simeq \pi_{0}\left(\mathscr{R}\left(\mathcal{O}_{1}\right)\right) \bar{\otimes} \pi_{0}\left(\mathscr{R}\left(\mathcal{O}_{2}\right)\right)$ is also a factor and from this it follows that $\pi_{p}$ is isomorphic to a subrepresentation of $\pi_{0}$. Hence by Lemma $2.1 \varphi_{p}$ can be extended to a vector state of $\mathscr{B}(\mathscr{H})$.

Thus it suffices in principle to calculate the norm distance of the states $\varphi_{0}$ and $\varphi_{p}$ with respect to $\mathscr{R}\left(\mathcal{O}_{1}\right) \vee \mathscr{R}\left(\mathcal{O}_{2}\right)$ in order to prove or disprove the existence of normal product states. Yet this is a very hard task even in the simple model which was the subject of this paper.

\section{Appendix A}

As was pointed out in Chapter III, our Condition iii) differs from the condition originally given by Araki [7; Lemma 6.5]. For the sake of completeness we want to prove the equivalence of both formulations.

Lemma. Let $0 \leqq S_{0} \leqq \mathbb{1}$ and $0 \leqq S_{p} \leqq \mathbb{1}$ be two operators which do not have the eigenvalue $\frac{1}{2}$ on $K_{0}(\boldsymbol{O})$ and $K_{p}(\boldsymbol{O})$ respectively. Assume furthermore that the norms $\|\cdot\|_{0}$ and $\|\cdot\|_{p}$ are equivalent. Then the following conditions are equivalent:

i) The operator $\mathbb{1}-\sigma\left(S_{p}\right) e^{-\chi\left(S_{p}\right)} e^{\chi\left(S_{0}\right)} \sigma\left(S_{0}\right)$ is of Hilbert-Schmidt class in $K_{0}(\boldsymbol{O})$.

ii) The operators $\left(S_{0}-S_{p}\right)\left(\mathbb{1}-2 S_{0}\right)^{-1}$ and $\sqrt{S_{0}\left(\mathbb{1}-S_{0}\right)}-\sqrt{S_{p}\left(\mathbb{1}-S_{p}\right)}$ are of Hilbert-Schmidt class in $K_{0}(\boldsymbol{O})$.

(Here we have defined $\sigma\left(S_{p}\right)=\left|2 S_{p}-\mathbb{1}\right| \cdot\left(2 S_{p}-\mathbb{1}\right)^{-1}$ and $\chi\left(S_{p}\right)$ $=\operatorname{Tanh}^{-1} 2 \sqrt{S_{p}\left(\mathbb{1}-S_{p}\right)}$; the absolute value and the positive square root have to be taken in $K_{p}(\boldsymbol{O})$. The operators $\sigma\left(S_{0}\right)$ and $\chi\left(S_{0}\right)$ are defined analogously.)

Proof. It follows from the definition of $\operatorname{Tanh}^{-1}$ (.) that

and

$$
\begin{aligned}
e^{-2 \chi\left(S_{p}\right)} & \left.=\left(\mathbb{1}+2 \sqrt{S_{p}\left(\mathbb{1}-S_{p}\right.}\right)\right)^{-1}\left(\mathbb{1}-2 \sqrt{S_{p}\left(\mathbb{1}-S_{p}\right.}\right) \\
& =\left(\sqrt{S_{p}}+\sqrt{\mathbb{1}-S_{p}}\right)^{-2}\left(\sqrt{S_{p}}-\sqrt{\mathbb{1}-S_{p}}\right)^{2}
\end{aligned}
$$

$$
e^{2 \chi\left(S_{0}\right)}=\left(\sqrt{S_{0}}+\sqrt{\mathbb{1}-S_{0}}\right)^{2}\left(\sqrt{S_{0}}-\sqrt{\mathbb{1}-S_{0}}\right)^{-2} \text {. }
$$


Thus the operator in Condition (i) can be rewritten:

(i')

$$
\begin{aligned}
& \mathbb{1}-\left(\sqrt{S_{p}}+\sqrt{\mathbb{1}-S_{p}}\right)^{-1}\left(\sqrt{S_{p}}-\sqrt{\mathbb{1}-S_{p}}\right)\left(\sqrt{S_{0}}+\sqrt{\mathbb{1}-S_{0}}\right) \\
& \cdot\left(\sqrt{S_{0}}-\sqrt{\mathbb{1}-S_{0}}\right)^{-1} .
\end{aligned}
$$

To begin with, let us show that $\sqrt{S_{0}\left(\mathbb{1}-S_{0}\right)}-\sqrt{S_{p}\left(\mathbb{1}-S_{p}\right)}$ is of HilbertSchmidt class if Condition (i) is satisfied. For this purpose we multiply the operator (i') on the left by $\sqrt{S_{p}}\left(\sqrt{S_{p}}+\sqrt{\mathbb{1}-S_{p}}\right)$ and on the right by $\left(\sqrt{S_{0}}-\sqrt{\mathbb{1}-S_{0}}\right) \sqrt{S_{0}}$. It follows then that

$$
\sqrt{S_{p}\left(\mathbb{1}-S_{p}\right)} \cdot S_{0}-S_{p} \cdot \sqrt{S_{0}\left(\mathbb{1}-S_{0}\right)}
$$

is of Hilbert-Schmidt class. If one multiplies ( $\left.i^{\prime}\right)$ on the left by $\sqrt{\mathbb{1}-S_{p}}\left(\sqrt{S_{p}}+\sqrt{\mathbb{1}-S_{p}}\right)$ and on the right by $\left(\sqrt{S_{0}}-\sqrt{\mathbb{1}-S_{0}}\right) \sqrt{\mathbb{1}-S_{0}}$ one can conclude that

$$
\left(\mathbb{1}-S_{p}\right) \sqrt{S_{0}\left(\mathbb{1}-S_{0}\right)}-\sqrt{S_{p}\left(\mathbb{1}-S_{p}\right)}\left(\mathbb{1}-S_{0}\right)
$$

is of Hilbert-Schmidt class too. The difference between (b) and (a) is just $\sqrt{S_{0}\left(\mathbb{1}-S_{0}\right)}-\sqrt{S_{p}\left(\mathbb{1}-S_{p}\right)}$. Hence this operator is of HilbertSchmidt class if Condition (i) is satisfied.

Since $\sqrt{S_{p}}+\sqrt{\mathbb{1}-S_{p}} \geqq \mathbb{1}$ in $K_{p}(\boldsymbol{O})$ and the norms $\|\cdot\|_{0},\|\cdot\|_{p}$ are equivalent it follows that $\sqrt{S_{p}}+\sqrt{\mathbb{1}-S_{p}}$ has a continuous inverse in $K_{0}(\boldsymbol{O})$. If one now multiplies (i') on the left by $\left(\sqrt{S_{p}}+\sqrt{\mathbb{1}-S_{p}}\right)^{2}$ and on the right by $\left(\sqrt{S_{0}}+\sqrt{\mathbb{1}-S_{0}}\right)^{-2}$ one gets after a simple calculation the result

$$
2\left\{\sqrt{S_{p}\left(\mathbb{1}-S_{p}\right)}-\sqrt{S_{0}\left(\mathbb{1}-S_{0}\right)}\right\}+2\left(S_{p}-S_{0}\right)\left(\mathbb{1}-2 S_{0}\right)^{-1}
$$

and this operator is of Hilbert-Schmidt class iff Condition (i) is satisfied. From this fact and the preceding considerations the statement of the lemma then follows immediately.

\section{Appendix B}

Lemma. Let $X, Y$ be bounded operators on a Hilbert space $\mathscr{H}$ such that $X \geqq 0, X^{2}+Y \geqq 0$. We furthermore assume that $Y$ is of trace class. Then $H=\sqrt{X^{2}+Y}-X$ is of Hilbert-Schmidt class.

Proof. i) First we shall prove that $H$ is a compact operator: the function $\sqrt{z}$ is continuous for $z \geqq 0$. Thus by the Weierstrass approximation theorem there exists for every $\varepsilon>0$ a polynomial $P_{n}(z)$ such that $\left|\sqrt{z}-P_{n}(z)\right|<\varepsilon$ for $z \in\left[0,\left\|X^{2}\right\|+\left\|X^{2}+Y\right\|\right]$. From this it follows that $\left\|X-P_{n}\left(X^{2}\right)\right\|<\varepsilon$ and $\left\|\sqrt{X^{2}+Y}-P_{n}\left(X^{2}+Y\right)\right\|<\varepsilon$. Now the operator $P_{n}\left(X^{2}+Y\right)-P_{n}\left(X^{2}\right)$ is compact because it is a finite sum of operators 
each of which contains the trace class operator $Y$ as a factor. Since $\left\|\left\{\sqrt{X^{2}+Y}-X\right\}-\left\{P_{n}\left(X^{2}+Y\right)-P_{n}\left(X^{2}\right)\right\}\right\|<2 \varepsilon H$ can be approximated in the norm topology by compact operators. Thus $H$ is a compact operator itself.

ii) Since $H$ is compact and selfadjoint there exists an orthonormal basis $\left\{\Phi_{n}\right\}$ in $\mathscr{H}$ which diagonalizes $H, H \Phi_{n}=\lambda_{n} \Phi_{n}$. Furthermore $(H+X)^{2}=X^{2}+Y$, hence $Y=H^{2}+X H+H X$. Taking matrix elements of this equation one gets

$$
\left|\left(\Phi_{n}, Y \Phi_{n}\right)\right|=\left|\lambda_{n}^{2}+2 \lambda_{n}\left(\Phi_{n}, X \Phi_{n}\right)\right|=\left|\lambda_{n}\right| \cdot\left|\left(\Phi_{n},\{H+2 X\} \Phi_{n}\right)\right| .
$$

From the definition of $H$ it follows immediately that $-(H+2 X) \leqq H$ $\leqq(H+2 X)$. Thus one gets $\left|\lambda_{n}\right| \leqq\left|\left(\Phi_{n},\{H+2 X\} \Phi_{n}\right)\right|$ hence $\left|\lambda_{n}\right|^{2}$ $\leqq\left|\left(\Phi_{n}, Y \Phi_{n}\right)\right|$. Since $Y$ is a trace class operator we can conclude that $\sum_{1}^{\infty}\left|\lambda_{n}\right|^{2}<\infty$ and this proves our statement.

Acknowledgements. I wish to thank Professor R. Haag for drawing my attention to this problem and Dr. M. Rinke for numerous helpful discussions during a period of collaboration on these questions. I am also indebted to Professor H. Araki and Dr. J. E. Roberts for fruitful remarks.

\section{References}

1. Roos, H.: Commun. math. Phys. 16, 238 (1970)

2. Reeh,H., Schlieder, S.: Nuovo Cimento 22, 1051 (1961)

Araki,H.: Lecture Notes, Part I, Zürich (1967)

3. Schlieder,S.: Commun. math. Phys. 13, 216 (1969)

4. Sakai,S.: $C^{*}$-Algebras and $W^{*}$-Algebras. Berlin-Heidelberg-New York: Springer 1971

5. Dixmier,J.: Les Algébras d'Opérateurs dans 1'Espaces Hilbertien. Paris: GauthierVillars 1969

6. Araki,H., Shiraishi, M.: Publ. RIMS Kyoto Univ. 7, 105 (1971)

7. Araki,H.: Publ. RIMS Kyoto Univ. 7, 121 (1971)

8. Araki, H.: Progr. of Theor. Phys. 32, 956 (1964)

Dell'Antonio, G. F.: Commun. math. Phys. 9, 81 (1968)

9. Glimm, J.R., Kadison, R.V.: Pacific Journ. Math. 10, 547 (1960)

Communicated by R. Haag

D. Buchholz

II. Institut für Theoretische Physik der Universität Hamburg D-2000 Hamburg, Luruper Chaussee 149

Federal Republic of Germany 